\title{
Prácticas de aprendizaje-servicio como escenarios de confluencia entre la educación escolar y social
}

\author{
Domingo Mayor Paredes *
}

Resumen. Este trabajo presenta los resultados de una investigación ${ }^{1}$ centrada en el análisis de las razones que sustentan la participación de múltiples agentes socioeducativos en los proyectos de Aprendizaje-Servicio, así como su influencia en la programación curricular de aula y en distintas dimensiones relacionadas con la mejora escolar y social. El estudio tomó como eje de indagación el proyecto implementado en un aula de primaria, situada en un contexto socialmente desfavorecido, y los efectos que se iban desencadenando durante la implementación del mismo². Para ello se utilizó el estudio de caso como metodología de la investigación. Los hallazgos alcanzados permiten constatar las diversas razones que sustentan la implicación de disímiles agentes en los proyectos, los impactos producidos en algunos ejes estructurales de la programación curricular y la articulación de un nuevo escenario educativo, conformado a partir de las conexiones establecidas entre la escuela y la comunidad.

Palabras clave: Aprendizaje-Servicio; mejora educativa y social, relación escuela-comunidad; programación curricular.

\section{PRÁTICAS DE APRENDIZAGEM EM SERVIÇO COMO CENÁRIOS DE CONFLUÊNCIA ENTRE A EDUCAÇÃO ESCOLAR E SOCIAL}

Resumo. Este artigo apresenta os resultados de uma pesquisa focada na análise das razões que sustentam a participação de múltiplos agentes sócio-educacionais nos projetos de Aprendizagem em Serviço, bem como sua influência na programação curricular da sala de aula e, em diferentes contextos, relacionadas à melhoria escolar e social. O estudo utilizou como ponto de indagação o projeto implementado em uma sala de aula primária, situada em um contexto socialmente desfavorecido, e os efeitos que foram sendo desencadeados durante a sua execução. Para essa finalidade, o estudo de caso foi utilizado como metodologia de pesquisa. Os resultados obtidos permitem constatar as diversas razões que sustentam o envolvimento de diferentes agentes nos projetos, os impactos produzidos em alguns eixos estruturais da programação curricular e a articulação de

*Facultad de Formación del Profesorado, Universidad de Extremadura, España.

${ }^{1}$ Dicho estudio forma parte de una Tesis Doctoral: El Aprendizaje-Servicio como práctica educativa que promueve relaciones colaborativas entre la escuela y la comunidad. Estudio de caso.

${ }^{2}$ El proyecto elegido como unidad de análisis forma parte del programa del Grupo de Investigación para la recuperación de espacios públicos -El Ingenio-, aprobado por la Dirección General de Innovación Educativa y Formación del Profesorado de la Junta de Andalucía. 
um novo cenário educacional, formado a partir das conexões estabelecidas entre a escola e a comunidade.

Palavras-chave: Aprendizagem em serviço; melhoria educacional e social; relação escola-comunidade; programação curricular.

SERVICE-LEARNING TRAINING AS SCENARIOS OF CONFLUENCE BETWEEN ACADEMIC AND SOCIAL EDUCATION

Abstract. This paper presents the results of an analysis of the reasons arguing for the participation of multiple socio-educational agents in Service-Learning projects, and their influence on classroom curricular programming in various dimensions related to academic and social improvement. The study's axis of enquiry was a project implemented in a primary school classroom located in a socially deprived context, and the effects triggered during its implementation. The research methodology for this was the case study. The findings demonstrated various reasons supporting the involvement of dissimilar agents in the projects, the impacts on some structural axes of curricular programming and articulation of a new educational scenario, based on the connections established between school and community.

Keywords: Service-learning; educational and social improvement; schoolcommunity relationship; curricular programming.

\section{INTRODUCCIÓN}

En las sociedades complejas y cambiantes del siglo XXI la noción mejora aplicada al escenario escolar sirve de justificación para el análisis y la producción de teorías, políticas y prácticas que focalizan su atención en distintas dimensiones que configuran la misma. En esta línea, podemos señalar que dicha temática ha sido utilizada para fundamentar una plétora de discursos relacionados con: las reformas educativas y las innovaciones implementadas en el interior de los dispositivos escolares (Carbonell, 2006; Fullan, 2007); los cambios en el curriculum (Gimeno, 2010); la formación de profesionales reflexivos (Schön, 1992); el desarrollo profesional (Imbernón, 2011); el trabajo por competencias y la introducción de las nuevas tecnologías de la información y la comunicación (Pérez, 2007); el desarrollo del capital profesional (Hargreaves y Fullan, 2014); la participación de los menores en los asuntos escolares (Fielding, 2011), etc.

Asimismo, se han ido produciendo estudios que recogían la emergencia de discursos y prácticas educativas originadas en los bordes de los sistemas escolares, dando lugar a la creación de nuevos conceptos (educación no formal, informal, educación a lo largo de la vida, etc.) para representar el universo educativo ${ }^{3}$. En este sentido, en los diversos informes internacionales

${ }^{3}$ El universo educativo está conformado por los distintos escenarios por donde circula la educación: formal, informal y no formal. En él se incluyen los diferentes dispositivos, agentes, temáticas, procedimientos, etc., con influencia en el desarrollo individual y social. 
liderados por Coombs y Admed (1975); Faure (1973) y Delors (1996) se reconocía que, a pesar de los progresos en el mundo de la educación formal, se seguían produciendo algunos desfases en la institución escolar relacionados con la creciente obsolescencia de los conocimientos que los programas transmitían, las inadaptaciones entre educación formal y mundo productivo, las desigualdades educativas entre los grupos y la escasa vinculación entre la escuela y la sociedad.

Por otra parte, en el ámbito social el significante mejora se puede concebir como el tránsito de perspectivas y prácticas sectoriales, parciales y disciplinares a propuestas de diseños más integrales, interdisciplinares, relacionales y transversales (Subirats, 2002) con el propósito de generar respuestas provisionales a las nuevas demandas de una sociedad atravesada por la incertidumbre, desigualdad e inestabilidad que producen los cambios acelerados en las distintas esferas de la vida.

En esta línea han ido aflorando marcos teóricos y prácticas socioeducativas $^{4}$ (Ciudades Educadoras, Planes de Desarrollo Comunitario, Investigación-Acción-Participativa, Planes Educativos de Entorno, Comunidades de Aprendizaje, proyectos de Aprendizaje-Servicio, etc.), encabalgadas entre la Pedagogía Escolar y la Pedagogía Social, que postulan la necesidad de situar la Educación en el ámbito comunitario, ampliando sus contornos más allá del espacio escolar con el propósito de promover sinergias para aprovechar el potencial educativo que encierran las comunidades físicas y virtuales, donde la escuela es considerada un nodo más del conjunto de nodos que conforman el tejido de las redes enmarañadas por donde transitan los individuos y grupos humanos a lo largo y ancho de su vida (Carbonell, 2006; Delors, 1996).

Esta nueva concepción del hecho educativo constituye uno de los ejes reguladores de las prácticas de Aprendizaje-Servicio (a partir de ahora ApS), entendidas como acciones formativas encaminadas a promover aprendizajes significativos, útiles y relevantes en contextos educativos expandidos (escuela-comunidad-universidad), donde el profesorado, el estudiantado y otros agentes socioeducativos van conformando una comunidad epistémica orientada a diseñar procesos formativos con la finalidad de dar respuestas a algunas de las necesidades reales y sentidas en el entorno inmediato.

${ }^{4}$ Las prácticas socioeducativas son acciones sociales implementadas desde una perspectiva educativa, es decir, orientadas al desarrollo de las capacidades de individuos y grupos humanos. En las mismas participan diversos agentes (educadores sociales, psicólogos sociales, trabajadores sociales, monitores, etc.) pertenecientes a diversos ámbitos del saber. 


\subsection{El aprendizaje-servicio, una práctica educativa medida por una doble intencionalidad: pedagógica y social}

Las huellas primitivas en el ámbito internacional del ApS como práctica pedagógica, son numerosas y provienen de contextos culturales muy diversos. Algunos ejemplos de ellas los podemos encontrar en el "Servicio Social mexicano, los proyectos de acción de las escuelas alemanas [...]" (Máximo, 2010, pp. 108-109).

Rastreando en los albores del siglo XX podemos identificar a diferentes autores, postulados teóricos y prácticas que configuran las distintas líneas de intersección o itinerarios que dibujan este universo en construcción. Diversos estudiosos (Máximo, 2010; Puig y Palos, 2006) plantean que los primeros antecedentes de esta metodología podemos encontrarlos en el surgimiento, a principios de siglo XX, de la corriente educativa liderada, entro otros, por Dewey y James. Dewey defendía el aprender haciendo, y James propuso el servicio civil a la comunidad en sustitución del servicio militar, dando lugar a las primeras experiencias estructuradas de servicio comunitario estudiantil. En la institución universitaria (Antioch College. EEUU) dirigida por Dewey, se inició en 1921 el programa de servicio comunitario, "considerado el pionero del aprendizaje-servicio" (Tapia, 2001, p.7). En dicha institución el estudiantado realizaba servicios en el campo de la salud, la educación y otros campos afines.

También las ideas del pedagogo brasileño Paolo Freire influyeron significativamente en los pioneros del ApS, tanto en EEUU -donde estuvo exiliado algunos años-, como en América Latina. En muchos de los planteamientos teóricos recogidos en algunas de sus obras (Pedagogía del oprimido; La educación como práctica de la libertad, etc.) se pueden apreciar las conexiones con las dimensiones estructurales del ApS, planteado desde una concepción fuerte (Díaz, 2006). Su concepto de praxis, como suma de reflexión y acción, está vinculado con el mapa conceptual del ApS. Para Freire (1983) la teoría separada de la práctica es puro verbalismo inoperante y la práctica separada de la teoría es activismo ciego. Asimismo ha sido influyente su concepción del hombre, del mundo y de la educación. En esta línea plantea que la educación debe servir a los hombres y mujeres para leer la realidad, insertándose cada vez con mayor conciencia en ella, para poder transformarla.

Por otro lado, el ApS, en su devenir sociohistórico, ha sido interpretado y materializado de múltiples formas como lo evidencian las diversas definiciones y prácticas halladas. Un estudio realizado por Kendal (1990 citado en Martínez-Odría, 2008, p. 629) encontró hasta 147 nociones diferentes en la literatura especializada para significar esta práctica educativa de naturaleza multidimensional (instituciones y agentes que intervienen, 
ámbitos donde se desarrolla, dimensiones pedagógicas que la constituyen, tipología de servicios, edades de los participantes, etc.) y multidisciplinar (pedagogía, psicología, sociología, etc.) que se viene implementando por múltiples agencias en diversos contextos socioculturales.

A pesar de las diferencias y similitudes que guarda con otras prácticas experienciales (trabajo de campo, iniciativas solidarias y servicios comunitarios), el estudio científico de la evolución de dicho fenómeno ha posibilitado "un consenso a nivel internacional que apunta a definir al aprendizaje-servicio a partir de tres dimensiones fundamentales" (Montes, Tapia y Yaber, 2011, p. 9): a) Se trata de un servicio a la comunidad con el propósito de dar una respuesta a necesidades reales y sentidas; b) Existe una planificación intencional e integrada de los contenidos curriculares y las actividades relacionadas que conforman el servicio a la comunidad; y c) Se promueve la participación fuerte del estudiantado en las distintas fases del proyecto: diseño, implementación y evaluación.

En el escenario iberoamericano se suele asumir la definición de ApS planteada por Puig y Palos (2006, p. 4): "Es una propuesta educativa que combina procesos de aprendizaje y de servicio a la comunidad en un solo proyecto bien articulado en el que los participantes se forman al trabajar sobre necesidades reales del entorno con el objetivo de mejorarlo".

Los proyectos de ApS se constituyen como actividades complejas que quiebran la forma de enseñanza de los dispositivos escolares tradicionales, basada en aprendizajes declarativos abstractos, descontextualizados y de escasa relevancia social. Esta forma de enseñar se traduce en aprendizajes poco significativos, relevantes y útiles, y en la incapacidad del alumnado para transferir y generalizar lo que aprenden a situaciones de la vida real. En cambio, en las acciones de ApS los aprendizajes, al estar imbricados con el servicio, favorecen la comprensión problemática de la realidad donde se va a actuar: diagnosticar y analizar las necesidades sociales a las que se pretende dar respuesta; implementar un servicio adecuado a dichas necesidades y reflexionar sobre lo acontecido. Todo ello favorece la significatividad, relevancia y utilidad de los aprendizajes, ya que son puestos en acción para clarificar y afrontar problemas básicos de la comunidad y para ampliar conocimientos, sensibilidades y afectos. En este sentido, lo que se aprende en las acciones de ApS se caracteriza por ser contextual, ya que parte de situaciones reales; por ser vivencial, porque requiere la implicación directa de los participantes en el proyecto; por ser integral, puesto que promueve el desarrollo de competencias necesarias para pensar y actuar sobre diversas realidades donde se ha decidido incidir y, por ser relacional, ya que requiere el enganche de diversas agencias educativas. 


\section{MÉTODO}

\subsection{Contexto de la investigación}

El proyecto de ApS, seleccionado como unidad de análisis, se implementó en un aula de $3^{\circ}$ de primaria, tenía como finalidad: mejorar la limpieza de algunas zonas del centro educativo y del barrio. Para ello se diseñó como una campaña de sensibilización en el centro educativo y en algunas zonas del barrio. En el mismo se integraron progresivamente distintas áreas curriculares: conocimiento del medio, lengua, matemáticas y artística. La selección de contenidos y los medios didácticos para abordarlos, las actividades dentro y fuera del aula, los horarios, etc., fueron objeto de negociación entre la docente y el estudiantado de primaria, contando con la colaboración del alumnado de la universidad, un grupo de familias, comerciantes, profesionales de distintos recursos públicos y la presidenta de la asociación de mayores.

Dicho programa pedagógico se desarrolló en un dispositivo escolar público, ubicado en una barriada de Almería catalogada por las instituciones públicas como zona en riesgo de exclusión social (Junta Andalucía, 2005), debido a los déficits identificados en distintos ámbitos: altos índices de abandono y fracaso escolar, escasa formación para acceder a los nuevos yacimientos de empleo, altas tasas de desempleo, problemas higiénico-sanitarios, número de infraviviendas, etc.) que afectan a la vida de la población que habita en el territorio. En esta zona urbana se vienen realizando actuaciones socioeducativas por parte de agencias públicas y entidades sociales con el propósito de mejorar la vida de la población. Una de esas acciones coordinadas fue la creación de un grupo de investigación ${ }^{5}$, constituido en el marco del Programa socioeducativo para la recuperación de espacios públicos "El Ingenio" 6 , donde se implicaron docentes del centro y profesoras y alumnado de la universidad, así como otros agentes socioeducativos de diferentes recursos públicos ubicados en el territorio (Empresa Pública de Vivienda, Centro de Salud, organizaciones sociales, etc.), con el doble propósito de, por un lado, implementar proyectos de ApS que favorecieran relaciones colaborativas entre escuela-comunidad y, por otro lado, promover la adquisición de aprendizajes útiles y relevantes dirigidos a la mejora escolar y social (Mayor, et al., 2012).

5 Dirección General de Innovación Educativa y Formación del Profesorado. Resolución de 26 de junio de 2012. BOJA núm. 157, p.54.

${ }^{6}$ Dicha acción socioeducativa se le concedió el primer premio de la la Edición concurso de proyectos de Educación Social "Memorial Toni Juliá", organizado por el Consejo General de Educadoras y Educadores Sociales (España). 


\subsection{Enfoque metodológico}

Con la intención de adentrarnos en el análisis de lo que dicen y hacen las personas, protagonistas en el proceso, en relación a sus propósitos, a las acciones implementadas y las mejoras producidas en cada uno de los contextos educativos (aula y comunidad), optamos por una metodología encuadrada dentro de la perspectiva interpretativa, ya que entendemos la educación como un fenómeno social complejo y la investigación como una oportunidad de interpretarla y mejorarla. Como estrategia de indagación se optó por el estudio de caso con el objetivo de identificar y comprender la complejidad del fenómeno objeto de estudio, su estructura dinámica, aquella que da razón plena de su comportamiento y manifestaciones (Simons, 2011), ya que interesaba conocer, desde la perspectiva de sus agentes, cómo se desarrollaba un proyecto de ApS en un contexto singular y su incidencia en diversas dimensiones vinculadas con la mejora escolar y social.

El proceso de indagación se estructuró en cuatro fases (preparatoria, trabajo de campo, analítica e informativa) que se interrelacionaban unas con otras, pero siempre en un camino hacia adelante en el intento de responder a las cuestiones planteadas en la investigación.

En el transcurso de la fase preparatoria se realizó el diseñó la investigación, contemplando las distintas dimensiones (unidad de análisis, proceso de selección de los participantes, compromisos y autorizaciones de los informantes, instrumentos de recogida de datos, temporalización, técnicas de análisis de datos, etc.). Paralelamente se fueron analizando los datos obtenidos, elaborando el pre-informe, negociando con los informantes, para concluir con la redacción del informe final (Rodríguez, Gil y García, 1999, p. 64).

\subsection{Selección del proyecto y participantes}

Para la selección del proyecto objeto de análisis se contemplaron algunos de los criterios que gozan de mayor consenso internacional, para significar un servicio de calidad a la comunidad: proyectos que ofrezcan un servicio orientado a mejorar algún aspecto de la realidad escolar y/o social, proyectos que favorezcan la conexión escuela-comunidad y proyectos que contemplen los objetivos curriculares previstos en el currículum oficial y el servicio a la comunidad (Montes, Tapia y Yaber, 2011).

Los informantes fueron las personas implicadas en la implementación del proyecto de ApS: 1 maestra y 17 alumnos de primaria, 1 profesora y 5 alumnos de la universidad, 1 representante de una entidad socia, personal del centro de salud y un grupo de familias y comerciantes. Para su 
selección se siguieron los siguientes criterios: disponibilidad e interés para colaborar en la investigación (Stake, 2010), así como el mayor tiempo de recorrido de la experiencia. En esta práctica de ApS, la docente, a diferencia de otras compañeras del centro que también desarrollaban proyectos durante un cuatrimestre, optó por implementarlo a lo largo de todo el curso escolar, lo cual favorecía un mayor tiempo de indagación del fenómeno de estudio.

\subsection{Instrumentos y procedimiento}

El proceso de indagación se realizó a lo largo de un curso escolar contando con la presencia del investigador en el centro educativo un día a la semana durante dos horas diarias, con la intencionalidad de recolectar los datos en el escenario donde acontecen los hechos, para lo cual se utilizaron las siguientes técnicas cualitativas:

a) El Análisis de documentos se focalizó en El Programa socioeducativo para la recuperación de El Ingenio, la Programación Curricular de Aula, Proyecto Educativo de Centro, Programa de la asignatura de Innovación Educativa, Diario de prácticas del alumnado de la Universidad, materiales didácticos elaborados por el estudiantado de primaria: libreta de mi vida, carteles, cartas, entrevistas, maquetas, etcétera.

b) La observación participante se desarrolló en el aula y en las distintas actividades que se realizaban en el centro educativo y en el barrio. Las informaciones obtenidas se fueron recogiendo en notas de campo que han constituido el diario de observación.

c) La entrevista semiestructurada grupal y la individual fueron diseñadas con el objetivo de contrastar algunas informaciones obtenidas durante el proceso de observación y análisis de documentos. Asimismo se utilizaron para ampliar algunos asuntos que afloraban durante la indagación. El guion abierto, que orientaban a las mismas, fue validado según el procedimiento de juicios de expertos para enfocar las cuestiones relevantes para la investigación. La entrevista individual se realizó a la maestra, profesora de la Facultad de CC. de la Educación de la Universidad de Almería y representante de una entidad social del barrio. La grupal fue dirigida alumnado de primaria y de la universidad. 
Con el propósito de intentar asegurar la credibilidad y replicabilidad de los datos (Guba, 1989) y eliminar el sesgo del investigador, las observaciones y las entrevistas efectuadas fueron grabadas en vídeo y, posteriormente, se realizó la transcripción literal de todo el material recogido, presentándose la misma a los informantes para obtener su validación.

\subsection{El proceso de análisis de los datos}

Para el estudio de los datos, se utilizó la técnica de análisis de contenido, cuyo propósito es clasificar, codificar y analizar el contenido y posteriormente construir las temáticas o categorías significativas (Simons, 2011). De dicho proceso emergieron múltiples categorías. En este estudio hemos seleccionado las vinculadas a la maestra y alumnado de primaria, a los cambios producidos en la programación curricular de aula y las mejoras producidas en la comunidad.

\section{TABLA 1}

\section{Categorías, subcategorías y códigos fijados en el análisis}

\begin{tabular}{|c|c|c|c|c|c|}
\hline Categorías & Códigos & Subcategorías & Códigos & Subcategorías & Códigos \\
\hline $\begin{array}{l}\text { Razones que } \\
\text { sustentan la } \\
\text { participación } \\
\text { de la maestra } \\
\text { y del alumnado } \\
\text { de primaria }\end{array}$ & RPA & $\begin{array}{l}\text { Mejora práctica profe- } \\
\text { sional. } \\
\text { Conexión aula-vida de } \\
\text { los menores. } \\
\text { Colaboración entre } \\
\text { agencias y agentes } \\
\text { educativos. }\end{array}$ & $\begin{array}{l}\text { RPAMP } \\
\text { RPAC } \\
\text { RPACA }\end{array}$ & $\begin{array}{l}\text { Aprendizajes diver- } \\
\text { tidos. } \\
\text { Nos gusta salir al } \\
\text { barrio. } \\
\text { Mejoramos nuestro } \\
\text { barrio. }\end{array}$ & $\begin{array}{l}\text { RPAAD } \\
\text { RPASB } \\
\text { RPAMB }\end{array}$ \\
\hline $\begin{array}{l}\text { Cambios en la } \\
\text { programación } \\
\text { aula }\end{array}$ & CPA & $\begin{array}{l}\text { Contextualización de } \\
\text { los contenidos. } \\
\text { Metodología. } \\
\text { Materiales didácticos. }\end{array}$ & $\begin{array}{l}\text { CPACC } \\
\text { CPAM } \\
\text { CPAMD }\end{array}$ & $\begin{array}{l}\text { Tiempos y espacios. } \\
\text { Relaciones. } \\
\text { Evaluación. } \\
\text { Participación me- } \\
\text { nores }\end{array}$ & $\begin{array}{l}\text { CPATE } \\
\text { CPAR } \\
\text { CPAE } \\
\text { CPAPM }\end{array}$ \\
\hline $\begin{array}{l}\text { El Servicio y } \\
\text { las relaciones } \\
\text { colaborativas }\end{array}$ & SRC & $\begin{array}{l}\text { Trabajo colaborativo } \\
\text { agencias y agentes. } \\
\text { Aprovechar el poten- } \\
\text { cial educativo de los } \\
\text { agentes. }\end{array}$ & $\begin{array}{l}\text { SRCTC } \\
\text { SRCPE }\end{array}$ & $\begin{array}{l}\text { Desarrollo de la co- } \\
\text { rresponsabilidad. } \\
\text { Mejora de la limpie- } \\
\text { za del barrio. }\end{array}$ & $\begin{array}{l}\text { SRCDC } \\
\text { SRCML }\end{array}$ \\
\hline
\end{tabular}

Fuente: Elaboración propia.

Con la intencionalidad de conseguir una información rigurosa se han seguido los criterios de confiabilidad propuestos por Guba (1989): credibilidad, dependencia, confirmabilidad y transferibilidad de la investigación. Para ello se han utilizado diversas estrategias: a) contextualización del objeto de indagación; b) registro audiovisual de la información obtenida; c) validación de las transcripciones por las personas implicadas; y d) triangulación entre los diversos instrumentos de recogida de información y entre las distintas fuentes 
utilizadas. Por lo tanto, la información presentada en el siguiente epígrafe es el resultado de examinar permanentemente los asuntos de interés que se iban obteniendo a través de los instrumentos de recogida de datos utilizados.

\section{RESULTADOS}

Los resultados de tipo cualitativo se expresan de manera narrativa, sistematizados en diversas categorías interrelacionadas, con descripciones de algunos de los asuntos más significativos que sostuvieron su configuración.

\subsection{Razones que sustentan el enganche de la maestra y el alumnado en los proyectos de $A p S$}

Los antecedentes del proyecto de ApS, descritos anteriormente, nos ayudan a situarnos en la atmósfera discursiva que, en este escenario complejo se iba expandiendo entre diversos agentes y dispositivos. Conceptos como integral, interdisciplinar, participación, trabajo colaborativo, potencial educativo de diferentes agentes e instituciones comunitarias, etc., se fueron instalando en el lenguaje común. También las primeras experiencias de trabajo conjunto y los resultados obtenidos fueron propiciando la emergencia de relaciones que transitaron por distintos niveles de enganche y se materializaron en diversas acciones. En este sentido, podríamos plantear que se fue generando un espacio y un tiempo donde lo posible pasó a formar parte de las representaciones sociales de los agentes. Un escenario que favoreció la concreción de nuevos proyectos que emergían a partir de la creación de espacios de intersección donde confluían diferentes cosmovisiones educativas. Se estaba produciendo lo que Laclau y Mouffe (1987) denominan como articulación: una práctica donde se establecen relaciones entre agentes de manera que sus identidades son modificadas como resultado de su inserción en la misma.

En línea con lo anterior, interesaba conocer las razones de distinta naturaleza que esgrimían los participantes del proyecto de ApS para involucrarse en una aventura que suponía cambios significativos en su quehacer profesional y estudiantil.

La maestra estaba interesada en "conectar la escuela con la vida de los menores" (Ent. M. ${ }^{7}$ ), por lo que su participación en el proyecto le posibilitó:

\footnotetext{
${ }^{7}$ Abreviaturas utilizadas: Entrevista Maestra (Ent. M.); Entrevista Alumnado
} (Ent. A.); Representante Entidad Social (R.E.S); Diario de observación (D.O). 
Conectar al colegio con el barrio y al barrio con el colegio. En este sentido no me siento que llego al centro y trabajo al margen de lo que me rodea. Para mí es estupendo que haya un grupo de profesionales de distintos ámbitos y alumnos de la universidad que permitan conectar la escuela y el barrio para enriquecerse mutuamente (Ent. M.).

Proyectar la mirada pedagógica más allá del aula le posibilitó vincularse con otros escenarios y agentes socioeducativos, hasta ese momento invisibles o no reconocidos, que podían colaborar en la consecución de los propósitos planteados en el proyecto de ApS. Las acciones colaborativas organizadas con los recursos y entidades sociales de la zona le sirvieron, por una parte, como marco referencial para ir repensando las razones, intenciones, temores y expectativas, potencialidades y dificultades iniciales y, por otra parte, para proyectar nuevas actividades en próximos cursos. Así lo manifestaba después de una sesión en el aula donde participaron un grupo de madres y padres junto a profesionales del centro de salud:

Para el próximo curso estoy pensando en realizar actividades con las familias para conocernos más. También he pensado en organizar alguna actividad con la Asociación de mayores, para que los mayores nos cuenten historias del barrio y los menores les canten canciones preparadas en clase (Ent. M.).

Otro aspecto significativo que le sirvió de impulso para implicarse en esta acción educativa es el valor otorgado a la dimensión social de la misma: "creo que el proyecto favorece a los menores implicarse activamente en la mejora de su entorno y, además, es otra forma de entender la educación, ya que se adapta más a las necesidades de este alumnado" (Ent. M.).

Por otro lado, los motivos que exponía el alumnado de primaria para engancharse en el proyecto no estaban dados de una vez y para siempre, fueron emergiendo y reconfigurándose acompañados de otras dimensiones del desarrollo humano conectadas a las expectativas, potencialidades, dificultades, condiciones materiales e institucionales, experiencias desarrolladas, aprendizajes adquiridos, significados atribuidos, etc. Así podemos encontrar que ante la pregunta genérica: ¿por qué has participado en el proyecto?, aparecen múltiples respuestas relacionadas con el tipo de actividades, el acompañamiento del alumnado de la universidad, las salidas al barrio, la diversión o el pasárselo bien, conocer a más gente, la pegada de carteles y las canciones, la tarta y la fiesta.

Entre las expresiones utilizadas por el estudiantado para representar su implicación en el proyecto aparecían de forma recurrente los vocablos diversión, gustaba y ayuda, junto a la frase aprendíamos y nos lo pasábamos bien: Porque nos hemos divertido y hemos aprendido (Ent. A.2). Porque no nos hemos aburrido y hemos estado trabajando (Ent. A.15). Porque nos 
gustaba salir al barrio a pegar carteles (Ent. A.6). Porque nos gustaban las actividades (Ent. A.4). Porque hicimos un Tipi y maquetas del río, y nos divertíamos (Ent. A.10).

Buscando comprender qué significa para el alumnado la noción aprendizaje divertido les pido que me concreten alguna actividad realizada en la que se lo hubieran pasado bien. La gran mayoría coincide con el día que salimos a ver el río y construimos el Tipi:

Son las 9,15 de la mañana. La maestra pide a los menores que cojan sus disfraces de la estantería y que se los vayan colocando. Se trata de una cinta con plumas que confeccionaron en la sesión anterior. Los menores están excitados, se ríen entre ellos al verse con las plumas en la cabeza. Se asoman al espejo y comentan cosas entre ellos. El alumnado de la universidad colabora en la colación de las cintas. Después ayudan a los menores a decorar sus caras con colores. Las risas siguen flotando en el ambiente. Ahora la maestra les recuerda que en el río van a recitar la frase que cada uno tiene asignada. Es un texto relacionado con una carta que un Gran Jefe Indio le envía al presidente de los Estados Unidos en defensa de sus tierras. Durante el recorrido la maestra y el alumnado de la universidad les preguntan a los menores por distintos objetos del barrio, y los menores responden sintiéndose importantes y reconocidos, ya que pueden hablar de algo que conocen. Cuando llegan al río el alumnado de la universidad desarrolla con ellos varios juegos de sensibilización medioambiental y graban un vídeo con las frases de la carta narradas por los menores. Después de una hora de intensa actividad, la maestra les pide que se sienten a desayunar. Antes de volver a clase, recogen los papeles de los bocadillos y algunas botellas y bolsas que estaban arrojadas en los alrededores. Cuando finaliza la recogida la docente plantea que si quieren construir una casa india en el aula como la que estuvieron dibujando unos días antes en el área de matemáticas. Se emocionan mucho: dicen que sí, gritan que sí, se ríen, gritan de alegría, y colaboran con la maestra en arrancar cuatro cañas que servirán como estructura de la casa. De regreso al colegio se encuentran una tabla de madera y la recogen para utilizarla como suelo del Tipi (D.O.). su memoria?

¿Cómo se producían los aprendizajes divertidos que guardan en

En la escena descrita, relacionada con el aprendizaje divertido, se pueden encontrar múltiples actividades de aprendizaje. En algunas de ellas, la maestra utilizaba la pregunta para que los menores hablasen de distintos aspectos de su barrio, "quería que ellos fueran protagonistas, que sintieran que podían ofrecer información a otros" (Ent. M.). En otras, el alumnado de la universidad y la maestra dirigían la actividad con la intencionalidad de que los menores adquiriesen nuevos conocimientos. Para ello utilizaban distintas temáticas y materiales y para su realización se requería poner en 
acción, además de capacidades cognitivas, capacidades afectivas, físicas y relacionales. Durante la implementación de las mismas el tiempo no era considerado con la rigidez que se le otorga en la organización estructurante de las áreas curriculares, sino que estaba en función del interés de la actividad.

A partir del análisis anterior, se desprende que la expresión de los menores relacionada con los aprendizajes divertidos, es una construcción compleja vinculada, entre otros asuntos, a momentos de aprendizaje que requieren del protagonismo fuerte del alumnado, la realización de diferentes actividades, la utilización de diversos materiales y una concepción del tiempo más flexible.

\subsection{Programación curricular y servicio a la comunidad: viajes de ida y vuelta mediados por la reflexión}

Después de rastrear las razones que sustentaban la participación de algunos agentes en las prácticas de A-S, focalizamos el análisis en la incidencia de dicha práctica en la programación curricular de aula. A sabiendas que es un recorte del proyecto como realidad construida colectivamente, nos decantamos por esta opción ya que nos permite tomar como eje de análisis a la profesional responsable de la planificación general del mismo, es decir, de su diseño, desarrollo y evaluación.

La programación didáctica o currículum en acción se configura, junto al diseño curricular y el proyecto educativo de centro, como uno de los dispositivos de ese objeto multidimensional y borroso denominado currículum.

Una invención reguladora del contenido y de las prácticas implicadas en los procesos de enseñanza-aprendizaje [...], que tiene capacidad para estructurar la escolarización, la vida de los centros educativos y las prácticas pedagógicas, pues dispone, transmite e impone reglas, normas y un orden que son determinantes (Gimeno, 2010, p. 26).

En este orden de ideas, la programación de aula supone un proceso de representación de la realidad de la vida en el aula, de los valores que se quieren transmitir, etc., así como una acción orientada a cambiar tal realidad. Dicha representación puede estar sustentada en presupuestos teóricos explícitos o implícitos, en rutinas establecidas dentro de la cultura escolar de un centro, en el conocimiento tácito construido en el ámbito de la experiencia, en las capacidades creativas y reflexivas de los docentes, en el deseo de cambio, etc. Dimensiones que se entrecruzan como manifestación de la complejidad del vivir humano y, por ende, del hecho educativo. 
En línea con lo anterior, la maestra manifestaba que después de varios años de experiencia en el centro había llegado a la conclusión de que en este contexto no se puede trabajar con una programación didáctica cerrada:

Cuando llegué el primer año tenía una estructura muy rígida, venía con mi programación cerrada para aplicarla. Y cuando llegué, los menores me tiraron la programación a la cara y me dijeron: "maestra, eso no vale". Estaba desconcertada, pensaba que si esa programación me había servido en otras escuelas como aquí no iba a servir (Ent. M.).

Su participación en el grupo de investigación, responsable de implementar proyectos de A-S en las distintas aulas del centro y su vinculación con dicha temática, la enfrentó a nuevos retos profesionales que supusieron novedosas formas de ir construyendo la programación de aula más cercanas a lo que Stenhouse (1984) considera como modelo proceso, que se basa en la idea de que son las experiencias de aprendizaje y el conocimiento relevante lo que hace valioso el currículum. En este sentido, la docente planteaba:

Este año tenía, por una parte, la programación formal relacionada con las distintas áreas curriculares y, por otra, el proyecto de A-S. Y en el transcurso del tiempo he ido fusionando los dos instrumentos de planificación. Por ejemplo: cuando estábamos haciendo la maqueta del río y en matemáticas estábamos viendo las figuras geométricas, y en conocimiento del medio el ciclo del agua, aprovechamos para relacionar dichos contenidos y creamos una piscina depuradora para colocarla en la maqueta del río que se estaba construyendo en el proyecto de ApS. A medida que íbamos viendo los contenidos de distintas áreas me di cuenta que podía integrarlos dentro del proyecto (Ent. M.).

Conectar la programación didáctica a los tres ejes estructurales que conforman las prácticas de ApS: vinculación de los objetivos curriculares y el servicio a la comunidad, la participación fuerte del alumnado en las distintas fases del proyecto y la concreción de actividades orientadas a promover alguna mejora en el ámbito comunitario, le sirvió, por un lado, para transitar por espacios conocidos y deseados relacionados con sus intereses y propósitos (enfoque creativo de los procesos de enseñanza-aprendizaje, vincular el aula con el barrio, etc.) y, por otro lado, para ir articulando un proyecto abierto y flexible que se verificaba en la acciones implementadas en el ámbito del aula y del barrio, posibilitando que los objetivos curriculares y las actividades derivadas del servicio a la comunidad se fueran retroalimentando mutuamente. Esta concepción del proceso de enseñanza-aprendizaje favoreció recoger la voz del alumnado de primaria durante las distintas fases del proyecto, flexibilizar los tiempos asignados a algunas actividades implementadas en el barrio, ir generando nuevas informaciones a partir de los interrogantes que se iban planteando en el transcurso de las actividades, así como abrir espacios para la participación del alumnado de la universidad y otros agentes socioeducativos. 


\subsection{El servicio como espacio de confluencia de saberes orientados} a la mejora de la realidad

En las prácticas de ApS, el servicio o actividad de utilidad social puede ser enfocado sobre distintos ámbitos, realizado por distintos grupos de población, implementarse en diferentes unidades de tiempo, favorecer la colaboración de diversos agentes, etc. Sobre él gravitan los contenidos y actividades de aprendizaje que emergen a partir de las conexiones que se establecen entre la escuela y la comunidad, entre agentes con influencia educativa en el medio donde se inserta el dispositivo escolar. En este sentido, el interés de la maestra orientado a que "Ios menores tuvieran información suficiente y pudieran sostener con más fuerza y razón el proyecto que nos habíamos planteado" (Ent. M.), la condujo a indagar en el territorio para detectar recursos y profesionales que pudieran enriquecer su trabajo. Se abría así un espacio de posibilidad para expandir el hecho educativo más allá de los límites de demarcación de la institución escolar, contemplando así el medio como contenedor o afluente de recursos educativos, como agente educativo que favorece el aprendizaje en distintos espacios comunitarios y como contenido de aprendizaje (Trilla, 2004).

Para resolver algunas de las dudas relacionadas con la campaña de sensibilización que estaban diseñando, la docente decidió establecer conexiones con diferentes profesionales:

Así, por ejemplo, para responder a la pregunta: “¿por qué algunas zonas del barrio están sucias?", creí conveniente contactar con la responsable de la empresa municipal de vivienda para que nos aclarará aspectos relacionados con la recogida y reciclaje de basura y otros enseres. Para contestar a otra de las cuestiones que nos habíamos formulado en la asamblea de clase: ¿cómo pueden influir la acumulación de basura en la salud?, nos pusimos en contacto con profesionales del centro de salud, y para conocer qué actividades se estaban haciendo en el barrio vinculadas con ese tema, contactamos con la presidenta de la asociación de mayores (Ent. M.).

Por otro lado, los diferentes agentes socioeducativos que participaron en el proyecto también se beneficiaron de esta relación educativa:

La enfermera me dijo que le parecía estupenda la actividad, y que aprovechando que tenían prácticos de enfermería en el centro aprovecharían esa actividad para que se dieran cuenta que el servicio que le podían prestar a la ciudadanía no era sólo el que se presta en el centro de salud, sino que se podían realizar acciones preventivas en el ámbito comunitario [...]. La responsable del Punto Limpio nos planteó que si los menores hacían la campaña de sensibilización en el barrio, ella se comprometía a instalar papeleras cerca del colegio [...]. Con la presidenta de la Asociación de 
mayores hemos quedado que para el próximo curso escolar vamos a organizar actividades para que ellas nos cuenten historias y juegos del barrio y nosotros preparamos canciones (Ent. M.).

\section{IMAGEN 1}

El alumnado de primaria entrevista a la responsable de la Empresa Municipal de Limpieza para conocer aspectos relacionados con la recogida de basura y el reciclaje de objetos

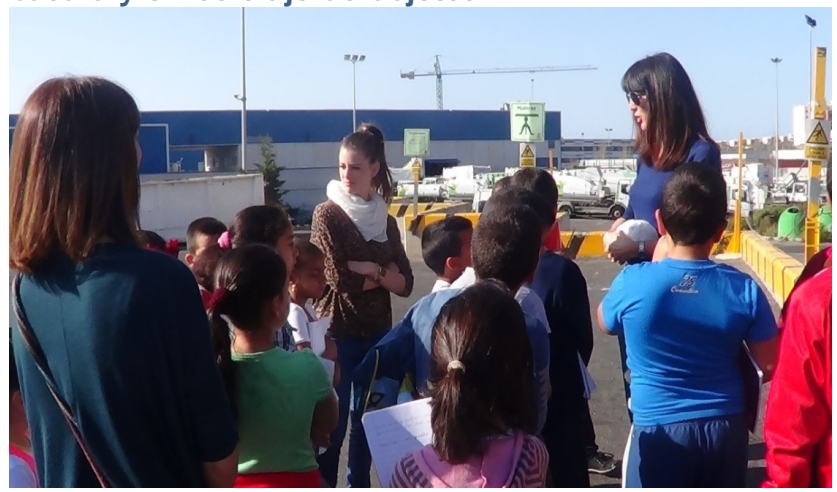

Fuente: Fotografía del autor (2014).

Los vínculos entre los distintos agentes se fueron ensanchando, pasando del conocimiento y reconocimiento a la colaboración, aprovechando el capital cultural instalado en el territorio para que el estudiantado adquiriera nuevos saberes con valor de uso. Un planteamiento próximo a los postulados de las Ciudades Educadoras y la nueva ecología del aprendizaje que sostienen que (Puig, 2009): la acción educativa está distribuida entre diferentes escenarios y agentes; la necesidad de un trabajo en el marco de la conformación de una red comunitaria integrada por los centros educativos, profesionales de los diferentes recursos públicos ubicados en la zona y representantes vecinales y de las administraciones públicas, que promueva coordinación de acciones formativas dirigidas a la población, favoreciendo así un dialogo de saberes procedentes de distintas disciplinas y la cultura popular, al objeto de airear los centros educativos, vincularlos más con el entorno y desentumecer la vida comunitaria; la necesidad de reconocer el rol educativo que pueden desempeñar los profesionales de diferentes recursos públicos y entidades sociales en los procesos formativos de los menores, y desde ese reconocimiento contemplar los aprendizajes que pueden adquirir los mismos en el desarrollo de la experiencia, etc. Aproximaciones que nos sitúan en la idea de que la educación de las nuevas generaciones es responsabilidad de todos los dispositivos y agentes insertos en la comunidad, entendida como red de agentes con influencia en la vida de menores y jóvenes. Como plantea 
Contreras (2006), uno de los aspectos centrales en la mejora de la educación escolar es el relacionado con el esfuerzo y la corresponsabilidad entre distintos agentes socioeducativos, dentro y fuera de los centros.

\section{CONCLUSIONES Y DISCUSIÓN}

\subsection{La reflexión sobre la práctica y las propuestas de mejora son ejes} articuladores que sustentan la implicación en las prácticas de ApS

Los hallazgos alcanzados en este estudio nos conducen a manifestar que las profesionales encargadas de la puesta en acción de las prácticas de ApS, desde una perspectiva fuerte, asumen un gran compromiso con la mejora de su práctica docente, situándose en lo que algunos autores consideran como profesionales reflexivas (Schön, 1992); innovadoras y/o comprometidas (Hargreaves y Fullan, 2014). Agentes que asumen su poder de cambio para ensayar con nuevos horizontes posibles que conllevan cambios significativos en algunas dimensiones de sus prácticas educativas.

Desde esta perspectiva, podemos plantear que los proyectos de ApS están configurados a partir del deseo de cambio de distintos agentes que se cuestionan, entre otros asuntos, su práctica profesional. Un deseo que responde a diversos intereses, expectativas, capacidades, etc., encontrando en el ApS un campo de experimentación para recrear nuevas formas de hacer que conectan con sus potencialidades actuales.

Podemos concluir exponiendo que las profesionales que apuestan por vincularse a proyectos de ApS manifiestan un interés inicial conectado a las ideas de mejora de su práctica profesional. Ideas sustentadas en dimensiones relacionadas con su desarrollo personal y profesional: interés por poner en acción nuevas metodologías, conocer nuevas formas de trabajo colaborativo, aprendizaje de nuevos conocimientos, ensayar propuestas que han ido elaborando en el tránsito por su experiencia, etc. También son agentes que se cuestionan su trabajo profesional y se revuelven hacia dentro para pensar en otras formas de hacer y relacionarse, donde la educación se piensa más allá de los márgenes de los dispositivos escolares para situarla en distintos espacios de intersección, en los cuales se producen múltiples vínculos entre diversos actores que influyen en el desarrollo del estudiantado y profesorado. 


\subsection{El ApS se configura como una instancia formativa para la mejora de diversas dimensiones de la programación curricular}

Atendiendo a los resultados alcanzados, se puede concluir que, en términos generales, los proyectos de A-S, en su versión fuerte (Díaz, 2006) o de alta calidad (Furco, 2007), tienen un impacto positivo en diversos elementos de la práctica docente: concepciones pedagógicas, diseño y puesta en acción de la programación didáctica y desarrollo profesional del profesorado.

En relación a la programación curricular, los resultados obtenidos nos permiten señalar que las prácticas de ApS se estructuran y funcionan como artefactos enmarañados que conllevan cambios sustantivos en diferentes elementos que integran la programación curricular de aula diseñada, en sus orígenes, desde una racionalidad instrumental o técnica. La interacción permanente entre los objetivos curriculares y los previstos en el servicio a la comunidad van conformando un círculo virtuoso (Montes et al., 2011), ya que los aprendizajes académicos mejoran el servicio ofrecido y el servicio estimula la adquisición o producción de nuevos conocimientos ("Durante el proceso descubrí que podía ir adaptando las distintas áreas curriculares al proyecto" -Ent. M-) para dar respuestas adecuadas a la necesidad detectada. En este sentido, y en consonancia con las aportaciones teóricas de diferentes autores (Pérez, 2004; Puig, 2009), esta investigación muestra la bondad pedagógica de los proyectos de ApS para configurar prácticas reflexivas y críticas, estructuradas por guiones curriculares flexibles donde los objetivos curriculares y los derivados del servicio a la comunidad van conformando una red de vasos comunicantes que se retroalimentan mutuamente.

De acuerdo con lo anteriormente expuesto, así como con algunas investigaciones realizadas (Díaz, 2006; Furco, 2007; Jouannet, Ponce y Contreras, 2012) y con las propuestas de mejora para transformar la educación que plantean distintos teóricos (Hargreaves y Fullan, 2014; Schön, 1992), consideramos que las prácticas pedagógicas de ApS pueden ser concebidas como escenarios formativos que promueven la mejora de diversas dimensiones de la práctica educativa.

\subsection{Situar la educación en el ámbito comunitario abre un horizonte de posibilidad para el trabajo colaborativo entre agentes que trabajan en el territorio}

Esta investigación arroja una conclusión muy evidente en relación a como el acercamiento interesado entre actores pertenecientes a distintos ámbitos educativos que reflexionan sobre cómo mejorar algunos aspectos de la realidad sociocultural y física de un territorio, genera una idea más 
expandida de la educación, en la cual los mapas educativos institucionales y profesionales se reconocen y/o amplían para convertirse en mapas territoriales o redes que crean artefactos inéditos hasta ese momento.

Durante las entrevistas realizadas pudimos observar el valor positivo asignado por la maestra, responsable del diseño e implementación del proyecto de ApS, a las producciones pedagógicas que se habían realizado. Asimismo, señalaba las bondades educativas de las actividades organizadas con la colaboración de profesionales del Centro de Salud, Empresa Municipal de Limpieza, representante de una entidad social de la zona y alumnado y profesora de la universidad. Acciones educativas realizadas dentro y fuera del aula que también fueron valoradas positivamente por el alumnado de primaria, ya que le permitió adoptar "un papel protagonista y reconstruir sus conocimientos" (Ent. M.). Igualmente sirvieron como marco de reflexiónacción para el alumnado y profesora de la universidad que observaba y participaba en otras formas novedosas de recrear el hecho educativo en el ámbito comunitario.

Estas potencialidades pedagógicas sirven para constatar el valor formativo de las propuestas de ApS, que al igual que otros modelos de educación expandida, como son las Ciudades Educadoras, las Comunidades de Aprendizaje o los modelos de Desarrollo Comunitario, promueven relaciones colaborativas y la adquisición de aprendizajes múltiples y multidireccionales (Carbonell, 2006; Puig, 2009; Trilla, 2004).

En relación a la prospectiva, se indican diversas líneas de investigación para profundizar en el estado de la cuestión de las mejoras producidas en el ámbito escolar y social, a partir de la implementación de proyectos de ApS:

a) Indagar la influencia que tienen las experiencias de ApS en el alumnado en distintas etapas escolares y en los agentes socioeducativos.

b) Estudiar el impacto de las prácticas de ApS en los cambios y mejoras producidas en el centro educativo y en la comunidad.

\section{REFERENCIAS BIBLIOGRÁFICAS}

Carbonell, J. (2006). La aventura de innovar. Madrid: Morata.

Coombs, P. H. y Ahmed, M. (1975). La lucha contra la pobreza rural. El aporte de la educación no formal. Madrid: Tecnos. 
Contreras, J. M. (2006). Compartir propósitos y responsabilidades para la mejora democrática de la educación. Revista de Educación, 339, pp. 19-41. Consultado el 30 de mayo de 2015. http://www.revistaeducacion.mec.es/re339/re339_03.pdf

Delors, J. (coord.). (1996). La educación encierra un tesoro. Madrid: Santillana.

Díaz, F. (2006). Enseñanza situada: vínculos entre la escuela y la vida. México: McGraw-Hill Interamericana.

Faure, E. (1973). Aprender a ser. Madrid: Alianza.

Fielding, M. (2011). La voz del alumnado y la inclusión educativa: una aproximación democrática radical para el aprendizaje intergeneracional. Revista Interuniversitaria de Formación del Profesorado, 70(25,1), 31-61.

Freire, P. (1983). La educación como práctica de la libertad. España: Siglo XXI.

Fullan, M. (2007). Las fuerzas del cambio con creces. Madrid: Akal.

Furco, A. (2007). Impactos de los proyectos de aprendizaje-servicio. En A. González (coord.). Antología 1997-2007. Seminarios Internacionales de Aprendizaje y Servicio Solidario, pp. 175-183. Buenos Aires: EUDEBA. Editorial Universitaria de Buenos Aires.

Gimeno, J. (Comp.). (2010). Saberes e incertidumbres sobre el currículum. Madrid: Morata.

Guba, E. (1989). Criterios de credibilidad en la investigación naturalista. En J. Gimeno y A. I. Pérez. (coords.). La enseñanza: su teoría y su práctica, pp. 148-165. Madrid: Akal.

Hargreaves, A. y Fullan, M. (2014). Capital profesional. Madrid: Morata.

Imbernón, F. (2011). Un nuevo desarrollo profesional del profesorado para una nueva educación. Revista de Ciencias Humanas, 12(19), pp. 75-86.

Jouannet, C., Ponce, C. y Contreras, A. (2012). Impacto de la metodología de AprendizajeServicio según la percepción de los docentes. En M. A. Herrero y M. N. Tapia (comps.). Actas de las /I Jornadas de Investigadores sobre Aprendizaje-Servicio. Buenos Aires: CLAYSS.

Junta de Andalucía (2005). Actuaciones en Barriadas y Zonas con Necesidades de Transformación Social. Consejería de Igualdad y Bienestar Social.

Laclau, E. y Mouffe, C. (1987). Hegemonía y estrategia socialista. Hacia una radicalización de la democracia. Madrid: Siglo XXI.

Martínez-Odría, A. (2008). Service-learning o aprendizaje-servicio. La apertura de la escuela a la comunidad local como propuesta de educación para la ciudadanía. Bordón, 59(4), pp. 627-640.

Mayor, D., García, T., Sánchez, M. S., Rodríguez, D., Zaballos, Mª. M., Pérez, I. y Romera, A. (2012). Programa socioeducativo de carácter comunitario para la recuperación de "El Ingenio-El Puche". En Consejo General de Colegios de Educadoras y Educadores Sociales. I Edición concurso de proyectos de Educación Social (pp. 13-59). Barcelona.

Máximo, E. (2010). Aprendizaje-Servicio en América Latina: apuntes sobre pasado y presente. Tzhoecoen Revista científica, 5, pp.108-125. Consultado el 2 de enero de 2015. http://creasfile.uahurtado.cl/TZHOECOEN.pdf 
Montes, R., Tapia, M., y Yaber, L. (2011). Manual para docentes y estudiantes solidarios. Buenos Aires: CLAYSS. Consultado el 5 de marzo de 2015. http://www.clayss.org. ar/04_publicaciones/Natura2013.pdf

Pérez, A. I. (2004). La cultura escolar en la sociedad neoliberal ( $4^{a}$ ed.). Madrid: Morata.

Pérez, A. I. (2007). La naturaleza de las competencias básicas y sus implicaciones pedagógicas. Cuadernos de Educación. Santander: Consejería de Educación de Cantabria.

Puig, J. M. (coord.). (2009). Aprendizaje Servicio. Educación y compromiso cívico. Barcelona: Graó.

Puig, J. M. y Palos, J. (2006). Rasgos pedagógicos del aprendizaje-servicio. Cuadernos de Pedagogía, 357, pp. 60-63.

Rodríguez, G., Gil, J. Y García, E. (1999). Metodología de la investigación cualitativa (2ª ed.). Málaga: Aljibe.

Schön, D. (1992). La formación de profesionales reflexivos. Hacia un nuevo diseño de la formación y el aprendizaje de las profesiones. Barcelona: Paidós.

Simons, H. (2011). El estudio de caso: Teoría y práctica. Madrid: Morata.

Stake, R.E. (2010). Investigación con estudio de casos. Madrid: Morata.

Subirats, J. (2002). El factor proximidad y de comunidad en las políticas educativas. En J. Subirats (coord.). Gobierno Local y Educación. La importancia del territorio y la comunidad en el papel de la escuela, pp. 21-50. Barcelona: Ariel.

Tapia, M. N. (2001). La solidaridad como pedagogía. El "aprendizaje-servicio" en la escuela. Buenos Aires: Editorial Ciudad Nueva.

Trilla, J. (2004). La educación no formal y la ciudad educadora. En H. Casanova y C. Lozano. Educación, universidad y sociedad: el vínculo crítico. Barcelona: Universidad de Barcelona. 
